\title{
ASSESSMENT OF METABOLIC MARKERS (MYOSTATIN, IGF-1), ASSOCIATED WITH THE STATE OF THE LEAN COMPONENT IN YOUNG ADULTS WITH TYPE 1 DIABETES
}

\section{DydyshkaY.V., Shepelkevich A.P.}

Department of Endocrinology, Belarusian State Medical University, Minsk, Belarus.

\section{BACKGROUND AND AIM:}

There is compelling evidence that the decline in appendicular muscle mass is a poor prognostic factor for the development of chronic complications of diabetes. In addition to chronic hyperglycemia, reduction of muscle component may be determined by metabolic disorders.

Therefore, the aim of study was to assess the possible links between serum myostatin, IGF-1 and a reduction in muscle mass in type 1 diabetes mellitus (T1DM).

SUBJECTS AND METHODS:

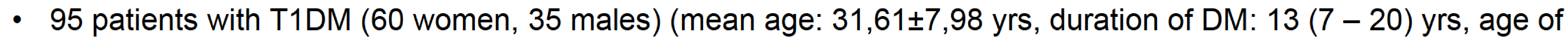

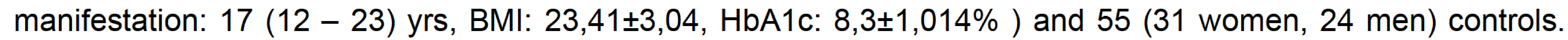

The research involved:

$\checkmark$ Anthropometry of patients ((height, weight, BMI, waist circumference)

$\checkmark$ General clinic examination

$\checkmark$ Biochemical analysis: HBA1, serum myostatin, IGF-1

RESULTS:

$\checkmark$ Dual-Energy X-ray Absorptiometry (DXA) using a program "Body composition"

$\checkmark$ There was a reduction of lean component of the $\operatorname{arms}(U=248 ; p=0,017)$, legs $(U=208 ; p=0,002)$, total appendicular $(U=219 ; p=0,004)$ and total lean $(U=259 ; p=0,027)$ component in men with T1DM.

$\checkmark$ Differences of lean mass at women with T1DM were in the increase of lean component of arms $(U=6774, p=0,044)$ and lean android $(U=604, p=0,008)$.

$\checkmark$ There were revealed comparable levels of serum myostatin in patients with T1DM and control groups, respectively 589 (457.26 - 826) and 675.38 (491.94 - 750.34) pg/mL; U=838; $\mathrm{p}=0.982)$ (Figure 1).

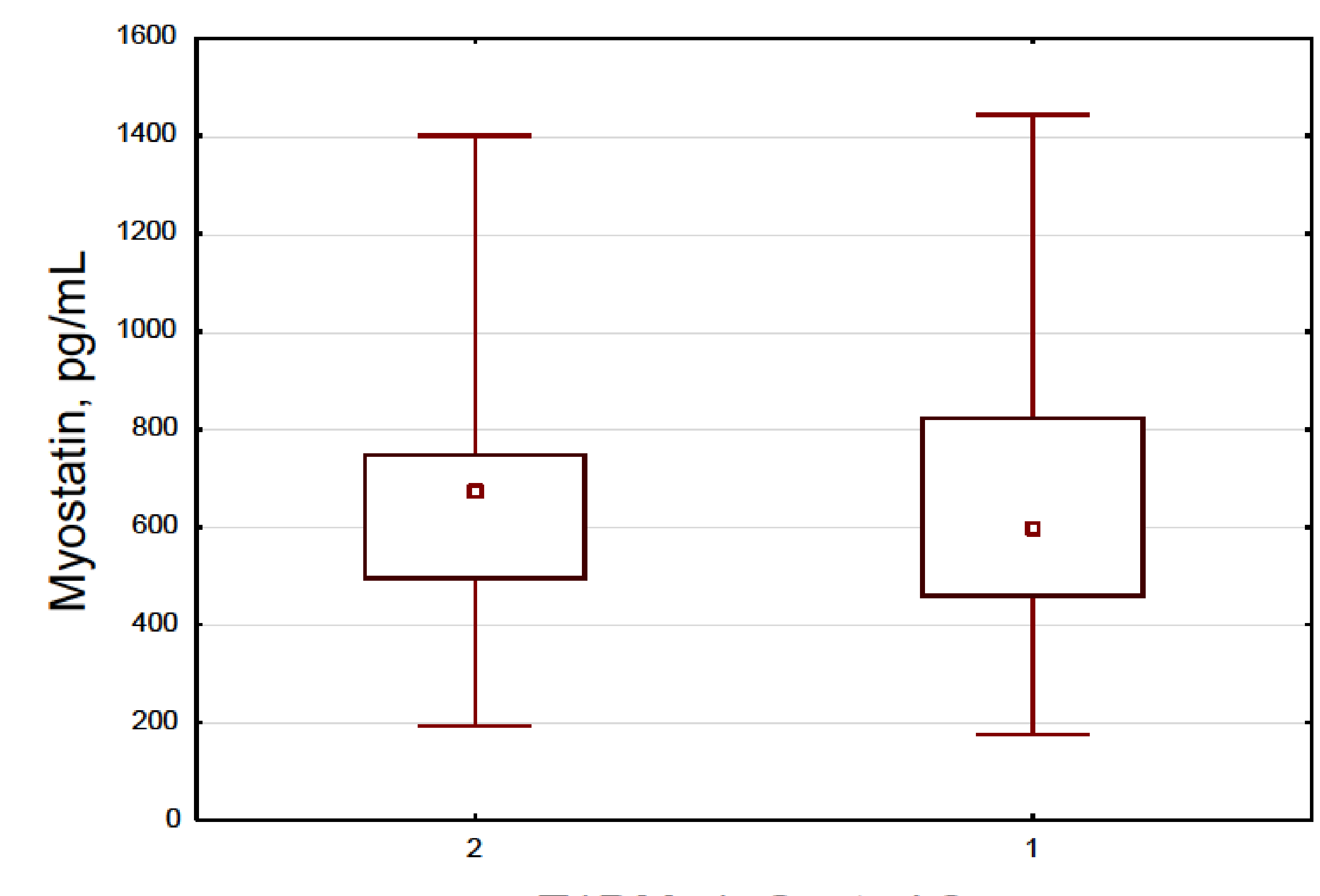

T1DM -1; Control-2

Figure 1 - Levels of serum myostatin in patients with T1DM and control groups

$\checkmark$. Men and women with T1DM have a comparable content of serum myostatin with control groups: women 529,23 $(455,65-625,86)$ vs $604,54 \quad(391,53-$ $745,53) \mathrm{pg} / \mathrm{ml}, \mathrm{U}=309 ; \mathrm{p}=0.585$; men 792,64 $(557,025-972,82) \quad$ vs $704,95 \quad(593,54-$ $790,23) \mathrm{pg} / \mathrm{mL}, \mathrm{U}=90 ; \mathrm{p}=0.48$, respectively. $\checkmark$ Taking into account gender differences the content of lean component, men with T1DM showed a significant and high levels of myostatin (792.64 (557.03 - 972.83) vs 529,23 $(443,55-625,86) \mathrm{pg} / \mathrm{mL}, \quad U=232,5 ; \mathrm{p}=0,006)$ compared to women that confirm the need for clinical interpretation of this indicator taking into account gender (Figure 2).

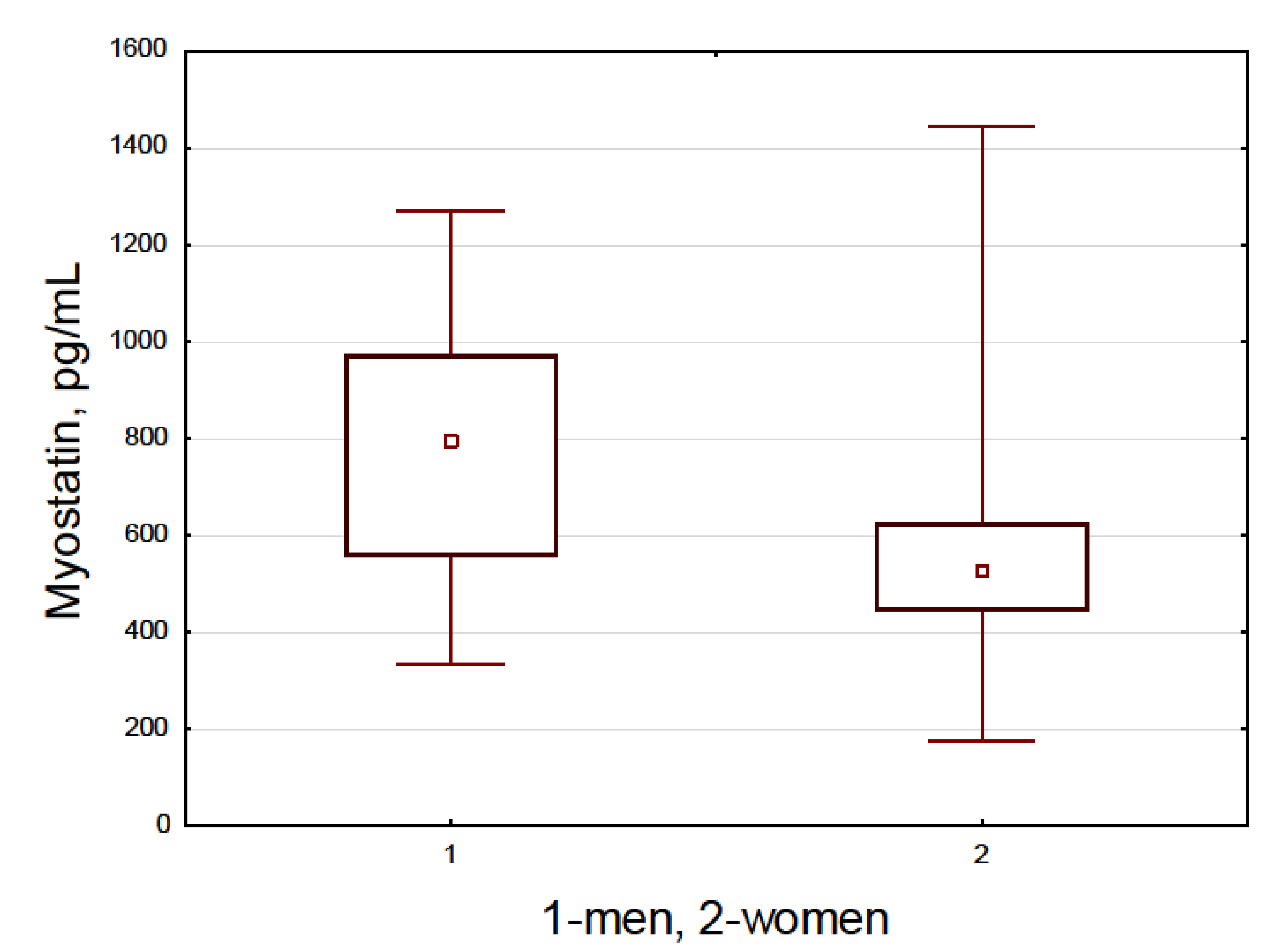

Figure 2 - Levels of serum myostatin in patients with T1DM

$\checkmark$ We were also obtained significant differences by gender in the content of serum IGF-1 in patients T1DM: men compared to women showed a significant lower content of IGF-1 (146,77 $(121,2-231,36)$ vs $106,15(96,28-138,67) \mathrm{ng} / \mathrm{ml}, \mathrm{U}=227,5$; $p=0,004)$.

\section{CONCLUSIONS:}

- Elevated levels of myostatin in men with diabetes can cause more expressed loss of muscle mass. Higher content IGF-1 explains the increase in the lean component of the abdomen in women. 